\title{
Optimization of a clamped plate silencer
}

\author{
Chunqi Wang, Jun Han, ${ }^{\text {a) }}$ and Lixi Huang ${ }^{\text {b) }}$ \\ Department of Mechanical Engineering, The Hong Kong Polytechnic University, Kowloon, Hong Kong
}

(Received 8 June 2006; revised 4 December 2006; accepted 5 December 2006)

\begin{abstract}
A previous theoretical study [L. Huang, J. Acoust. Soc. Am. 119, 2628-2638 (2006)] shows that, in a duct, a simply supported plate covering a side-branch rigid cavity can function effectively as a wave reflector over a broad range of low to medium frequencies. In this study, analytical formulation is extended to the boundary condition of clamped plate, which is easier to implement in practice. The theoretical model is tested experimentally using balsawood, which has a very high stiffness to mass ratio. The spectral peaks and shapes of the measured TL are in agreement with those calculated theoretically, attempts are also made to account for the considerable sound absorption in the rig. Further numerical studies based on the validated model show that, for a uniform plate, the optimal stopband is narrower and the lower band limit is worse than that of the simply supported configuration. However, a wave reflector using nonuniform, clamped plates with thinner ends out-performs the simply supported configuration in every aspect. Analyses show that the improvement is attributed to the increased acoustic radiation efficiency over the bulk length of the nonuniform plate, which behaves more like a rigid plate. (C) 2007 Acoustical Society of America. [DOI: 10.1121/1.2427126]
\end{abstract}

PACS number(s): 43.50.Gf, 43.20.Tb, 43.20.Ks [KA] Pages: 949-960

\section{INTRODUCTION}

Low frequency duct noise is difficult to deal with by traditional methods such as porous duct lining and expansion chamber. Lining a duct with porous sound absorbing material is a very mature and reliable technique that can tackle the medium to high frequency noise easily (Mechel and Vér, 1992; Ingard, 1994). However, it does not work well for very low frequencies. An expansion-chamber-type muffler may be used to reflect the low frequency noise, but such a device is usually bulky and passbands exist. Aiming for a broadband passive noise control device that works effectively in the low-to-medium frequency range, Huang (2002) introduced the concept of a drumlike silencer. It consists of an expansion chamber with two side-branch cavities covered by light membranes under a fairly high tension. The predicted noise reduction performance has been verified without flow (Choy and Huang, 2002) and with flow (Choy and Huang, 2005). In addition to the broad bandwidth in the low frequency region, such a device has two other merits. First, it is fiber-free, hence more environmentally friendly than the porous duct lining. Second, because the membranes are flush-mounted with the duct and there is no sudden change of duct area, the flow can pass the silencer smoothly without causing any extra noticeable pressure loss.

In a recent study, Huang (2006) replaced the membranes used in the drumlike silencer described above by a simply supported plate (simply supported plate silencer, hereinafter). Theoretical study has shown that the proposed plate silencer can achieve a much wider logarithmic bandwidth than the

\footnotetext{
${ }^{a}$ Current address: Ray W. Herrick Laboratories, Purdue University, 140 S. Intramural Drive, West Lafayette, IN 47907-2031.

${ }^{b)}$ Corresponding author. Current address: Dept. of Mechanical Engineering, The University of Hong Kong, Pokfulam Road, Hong Kong. Electronic mail: lixi.huang@hku.hk
}

drumlike silencer of the same cavity geometry due to the changed intermodal acoustic interference between the odd and even in vacuo vibration modes. Besides, the plate silencer carries other advantages over the drumlike silencer. As discussed in the study of Choy and Huang (2002), the optimal tension plays an important role in achieving the desired performance. However, it is not an easy job to tune or to maintain the tension at the optimal value in practice, and the tension may vary due to membrane relaxation or the change of ambient temperature. The plate uses its natural bending moment as the sole structural restoring force. Thus, both the installation and the maintenance of the plate silencer are simplified.

The use of a plate or the tensioned membrane in noise abatement may not be new, but its use as a side-branch wave reflector is. For example, panel absorbers were used in broadcasting studios and other architectural practices (Brown, 1964; Ford and McCormick, 1969; Sakagami et al., 1996; Horoshenkov and Sakagami, 2001); membrane absorbers were also used as a splitter silencer in the form of arrays of Helmholtz resonators (Frommhold et al., 1994). However, in all these applications, the panel, or membrane, is a component of resonator, which works for a narrow frequency band, and the structural mass is a means to achieving a low resonance frequency. For the plate silencer, the most desirable properties of the plate are the high stiffness and low density, which contrast with the characteristics of the existing use of plate in noise control.

In this study, the leading and trailing edges of the plate are clamped instead of simply supported, which leads to a fixed-end plate silencer, or clamped plate silencer. The motivation for this change is given as follows. The simply supported ends described in Huang (2006) can be easily modeled in theoretical study and a model with such boundary conditions can be conveniently analyzed as well. However, it 
is usually difficult to implement such a boundary condition in an acoustic system, especially in a system like the plate silencer discussed here. One reason is that the elements in the acoustic system are usually so light in mass that the methods to implement the simply supported ends in a real device often result in effective clamped ends. Hence, it represents a step forward to replace the ideal simply supported boundary conditions by a more practical one. During the course of this study, it is found that when the bending stiffness and mass distribution of the clamped plate are constant along the length of the plate (a uniform plate, hereinafter), the stopband of the plate silencer turns out to be narrower than that of a simply supported one. One qualitative explanation is that the clamped-clamped boundary condition reduces the effective radiation length of the plate when it vibrates in response to the incident sound. To release the additional restrictions caused by the clamped ends, it is proposed to use a plate with variable bending stiffness and mass distribution along the length of the plate, namely, a nonuniform plate. The problem of sound scattering of similar structures has been studied before. For example, Fernyhough and Evans (1996) compared a step approximation to an exact solution of acoustic scattering in an acoustic waveguide with nonuniform wall impedance; Grant and Lawrie (2000) studied the acoustic scattering by a duct in which the bending stiffness varies smoothly with distance along the plate. Huang (2001) also presented a two-dimensional theoretical analysis of a membrane of varying compliance in the passive control of duct noise. As far as the current study is concerned, the employment of the nonuniform plate is mainly motivated by reducing the restrictions due to the clamped ends and thus increasing the radiation length of the plate. Hence, a nonuniform plate with two softer ends is preferred.

In what follows, Sec. II outlines the analytical formulation for the clamped plate silencer with both uniform and nonuniform plate. The transmission loss (TL) of the clamped plate silencer with uniform plate is first examined and optimized in Sec. III. It shows that the performance of such a plate silencer is a little better than that of the drumlike silencer of the same geometry but not as good as that of the simply supported plate silencer. Then the optimization of the nonuniform plate is conducted and the results show that, with a proper distribution of bending stiffness along the plate, the clamped nonuniform plate performs better than the simply supported plate. In Sec. IV, the basic theoretical model for the clamped plate silencer is validated experimentally.

\section{ANALYTICAL FORMULATION}

\section{A. The theoretical model}

Figure 1 shows the two-dimensional configuration of a side-branch plate silencer. It has a two dimensional duct of height $h^{*}$, with two plates (beams) flush-mounted on the wall. The asterisks denote dimensional variables while the corresponding dimensionless ones are introduced shortly without asterisks. The leading and trailing edges of the two plates are clamped to the rigid duct at the two edges of $\left|x^{*}\right|=L^{*} / 2$, respectively, and are backed by a rigid-walled



FIG. 1. Theoretical model of sound wave reflection by two cavity-backed plates forming part of the otherwise rigid duct walls.

cavity of depth $h_{c}^{*}$. The two lateral edges of the plates are set free and the three-dimensional plate is reduced to a twodimensional beam. A plane incident wave comes from the left-hand side of the duct with a unit amplitude

$$
p_{i}^{*}=\exp \left[i\left(\omega^{*} t^{*}-k_{0}^{*} x^{*}\right)\right]
$$

and it causes the plate to vibrate with a transverse displacement of complex amplitude $\eta^{*}(x)$ and velocity $V^{*}(x)$ with the same time dependence, $\exp \left(i \omega^{*} t^{*}\right)$, which is henceforth omitted. The vibration radiates sound and imposes a radiation pressure on the plate surface. The problems of acoustics and plate vibration are fully coupled.

Before quantifying the plate dynamics, all variables are nondimensionalized as follows by three basic quantities, air density $\rho_{0}^{*}$, duct height $h^{*}$, and speed of sound in free space $c_{0}^{*}$ :

$$
\begin{aligned}
& x=\frac{x^{*}}{h^{*}}, \quad y=\frac{y^{*}}{h^{*}}, \quad t=\frac{c_{0}^{*} t^{*}}{h^{*}}, \quad L=\frac{L^{*}}{h^{*}}, \quad \eta=\frac{\eta^{*}}{h^{*}}, \\
& f=\frac{h^{*} f^{*}}{c_{0}^{*}}, \quad \omega=k_{0}=2 \pi f, \\
& p=\frac{p^{*}}{\rho_{0}^{*}\left(c_{0}^{*}\right)^{2}}, \quad m=\frac{m^{*}}{\rho_{0}^{*} h^{*}}, \quad T=\frac{T^{*}}{\rho_{0}^{*}\left(c_{0}^{*} h^{*}\right)^{2}}, \\
& B=\frac{B^{*}}{\rho_{0}^{*}\left(c_{0}^{*}\right)^{2}\left(h^{*}\right)^{3}} .
\end{aligned}
$$

Here, $m$ is the plate-to-air mass ratio, $B$ is the bending stiffness of the plate, which may vary with $x$, and $T$ is the tension of membrane. According to the above normalization scheme, the dimensionless first cut-on frequency of the rigid-walled duct is $f=0.5$. Other variables will be explained when they are used.

Assume that the bending stiffness $B$ and mass ratio $m$ are functions of the coordinate along the plate, that is, $x$. The dynamics of the lower plate vibration is governed by

$$
\frac{\partial^{2}}{\partial x^{2}}\left(B(x) \frac{\partial^{2} \eta}{\partial x^{2}}\right)+m(x) \frac{\partial^{2} \eta}{\partial t^{2}}+\left(p_{i}+\Delta p\right)=0
$$

where $p_{i}$ is the dimensionless incident wave and $\Delta p=p_{+}$ $-p_{-}$is the fluid loading acting on the upper $(+)$and lower $(-)$ sides of the plate induced by the plate vibration itself. When a uniform plate is used, bending stiffness $B$ and mass 
ratio $m$ are then constant along the plate, and Eq. (3) is simplified as

$$
B \frac{\partial^{4} \eta}{\partial x^{4}}+m \frac{\partial^{2} \eta}{\partial t^{2}}+\left(p_{i}+\Delta p\right)=0 .
$$

Note that the effect of damping is excluded in the theoretical studies since sound reflection is dominant in the plate silencer. The effect of damping that necessarily exists in experiment is modeled and discussed in Sec. IV.

\section{B. Modal dynamics of the sound-plate interaction}

For harmonic vibration, the plate normal vibration velocity is $V=\partial \eta / \partial t=i \omega \eta$. Equation (3) then becomes

$$
\frac{1}{i \omega}\left[\frac{\partial^{2}}{\partial x^{2}}\left(B(x) \frac{\partial^{2} V}{\partial x^{2}}\right)\right]+m(x) i \omega \cdot V+\left(p_{i}+\Delta p\right)=0 .
$$

The vibration mode shapes of a uniform clamped beam are available in textbooks such as (Inman, 2001) and, by introducing local variable $\xi$ below, it is rewritten as

$$
\begin{aligned}
\varphi_{j}(\xi)= & A_{1, j} e^{\beta_{j} \xi}+A_{2, j} e^{-\beta_{j} \xi}+A_{3, j} \sin \left(\beta_{j} \xi\right) \\
& +A_{4, j} \cos \left(\beta_{j} \xi\right), \quad \xi=(x / L+1 / 2),
\end{aligned}
$$

where

$A_{1, j}=\frac{1}{2}\left(1-\sigma_{j}\right), \quad A_{2, j}=\frac{1}{2}\left(1+\sigma_{j}\right), \quad A_{3, j}=\sigma_{j}, \quad A_{4, j}=-1$,

$\sigma_{j}=\frac{\cosh \left(\beta_{j}\right)-\cos \left(\beta_{j}\right)}{\sinh \left(\beta_{j}\right)-\sin \left(\beta_{j}\right)}, \quad \tan \left(\beta_{j}\right)+\tanh \left(\beta_{j}\right)=0$.

The coupled dynamics Eq. (5) can be solved via the standard Galerkin procedure, in which $V$ is expanded as a series of in vacuo modes $\varphi_{j}(\xi)$ of the clamped-clamped uniform plate with modal amplitudes $V_{j}$ :

$$
V=\sum_{j=1}^{\infty} V_{j} \varphi_{j}(\xi) .
$$

Equation (5) is then transformed as

$$
\sum_{l=1}^{\infty} V_{l} L_{j l}+\int_{0}^{1}\left(p_{i}+\Delta p\right) \varphi_{j}(\xi) d \xi=0,
$$

where the dynamic matrix $L_{j l}$ is given by

$$
\begin{aligned}
L_{j l}= & \int_{0}^{1}\left\{\frac { 1 } { i \omega } \frac { 1 } { L ^ { 4 } } \left[B(\xi) \varphi_{l}^{(4)}(\xi)+2 B^{(1)}(\xi) \varphi_{l}^{(3)}(\xi)\right.\right. \\
& \left.\left.+B^{(2)}(\xi) \varphi_{l}^{(2)}(\xi)\right]+i \omega m(\xi) \varphi_{l}(\xi)\right\} \varphi_{j}(\xi) d \xi .
\end{aligned}
$$

When a uniform plate is used, the dynamic matrix $\left[L_{j l}\right]$ reduces to a diagonal matrix with element

$$
L_{j j}=m i \omega+\frac{B}{i \omega}\left(\frac{j \pi}{L}\right)^{4} .
$$

In order to solve Eq. (9), the fluid loading $\left(p_{i}+\Delta p\right)$ has to be related to the modal vibration velocity amplitude $V_{j}$. In other words, a modal impedance $Z_{j l}$ must be found, $Z_{j l}$ being defined as

$$
Z_{j l}=\int_{0}^{1} \varphi_{l}(\xi) \Delta p_{j}(x) d \xi
$$

where $\Delta p_{j}(x)$ is the fluid loading caused by the $j$ th modal vibration of unit amplitude. A detailed method of solution to determine the modal impedance of the drumlike silencer was given in Huang (2002). The same procedure is followed here. Issues unique to the current model are (1) the mode $\sin (j \pi \xi)$ is replaced by $\varphi_{j}(\xi)$ defined in Eqs. (6) and (2) the fluid loading inside the cavity is found by the cavity modes to simplify the formulation. As said before, $p_{i}$ is the incident wave, and the fluid loading induced by the plate vibration, $\Delta p$, may be divided into two parts:

$$
\Delta p=p_{\text {rad }}-p_{\text {cav }},
$$

where $p_{+\mathrm{rad}}$ is the radiation sound pressure in the main duct and $p_{\text {cav }}$ is the pressure inside the cavity acting on the lower plate surface. The formulation for $p_{\text {+rad }}$ is well known (Doak, 1973), and is rewritten in 2D dimensionless form

$$
\begin{aligned}
p_{+\mathrm{rad}}(x, y)= & \frac{L}{2} \sum_{n=0}^{\infty} c_{n} \psi_{n}(y) \int_{0}^{1} \psi_{n}\left(y^{\prime}\right) V\left(x^{\prime}\right) \\
& \times\left[H\left(x-x^{\prime}\right) e^{-i k_{n}\left(x-x^{\prime}\right)}+H\left(x^{\prime}-x\right) e^{+i k_{n}\left(x-x^{\prime}\right)}\right] d \xi^{\prime},
\end{aligned}
$$

where $H$ is the Heaviside function, $c_{n}, k_{n}$, and $\psi_{n}$ are, respectively, the modal phase speed, the modal wave number, and the modal velocity potential:

$$
c_{n}=\frac{i}{\sqrt{(n \pi / \omega)^{2}-1}}, \quad k_{n}=\frac{\omega}{c_{n}}, \quad \psi_{n}(y)=\sqrt{2-\delta_{0 n}} \cos (n \pi y) ;
$$

and $\delta_{0 n}$ is the Kronecker delta. Hence, suppose the radiation pressure caused by the $j$ th modal vibration of unit amplitude $\varphi_{j}$ is $p_{+\mathrm{rad}, j}$. Then the modal impedance contributed by the radiation pressure in the main duct is defined as follows,

$$
\begin{aligned}
Z_{+j l} & =\int_{0}^{1} \varphi_{l}(\xi) p_{+\mathrm{rad}, j}(x, 0) d \xi \\
& =\frac{L}{2} \sum_{n=0}^{\infty} c_{n}\left(2-\delta_{0 n}\right) \sum_{p=1}^{4} \sum_{q=1}^{4} A_{p, l} A_{q, j} I_{p, q},
\end{aligned}
$$

where $A_{q, j}$ and $A_{p, l}$ are the coefficients defined in Eq. (7) and $I_{1,1}-I_{4,4}$ are given in the Appendix .

The acoustic pressure inside a lightly damped cavity can be expressed in terms of "rigid wall" modes of the cavity (Kuttruff, 2000). For the two-dimensional configuration considered here, the normalized pressure inside the cavity $p_{\text {cav }}$ can be written as

$$
\begin{aligned}
p_{\text {cav }}(x, y)= & \sum_{m, n} \frac{-i \omega \phi_{m, n}(x, y)}{L h_{c}\left(\kappa_{m, n}^{2}-k^{2}+2 i \zeta_{m, n} \kappa_{m, n} k\right)} \\
& \times \int_{0}^{1} V\left(x^{\prime}, 0\right) \phi_{m, n}\left(x^{\prime}, 0\right) d \xi^{\prime},
\end{aligned}
$$

where $V\left(x^{\prime}, 0\right)$ is the normal velocity over the flexible panel, 
$\zeta_{m, n}$ is the damping ratio of the $(m, n)$ th acoustic mode $\phi_{m, n}(x, y), L$ is the length of the cavity, $h_{c}$ is the height of the cavity, and $\kappa_{m, n}$ is the corresponding acoustic wave number of the $(m, n)$ th acoustic mode $\phi_{m, n}(x, y)$, with $\phi_{m, n}(x, y)$ and $\kappa_{m, n}$ given as

$$
\begin{aligned}
& \phi_{m, n}(x, y)=\sqrt{\left(2-\delta_{0 m}\right)\left(2-\delta_{0 n}\right)} \cos \left(\frac{m \pi x}{L}\right) \cos \left(\frac{n \pi y}{h_{c}}\right), \\
& \kappa_{m, n}^{2}=\left(\frac{m \pi}{L}\right)^{2}+\left(\frac{n \pi}{h_{c}}\right)^{2} .
\end{aligned}
$$

Therefore, the cavity pressure caused by the $j$ th modal vibration of unit amplitude, $p_{\mathrm{cav}, j}(x, y)$, can be found and the cavity modal impedance becomes

$$
\begin{aligned}
Z_{c a v, j l}= & -\int_{0}^{1} \varphi_{l}(\xi) p_{c a v, j}(x, 0) d \xi \\
= & \sum_{m=0}^{\infty} \sum_{n=0}^{\infty} \frac{i k\left(2-\delta_{0 m}\right)\left(2-\delta_{0 n}\right)}{h_{c}\left(\kappa_{m, n}^{2}-k^{2}+2 i \zeta_{m, n} \kappa_{m, n} k\right)} \\
& \times\left(\sum_{p=1}^{4} A_{p, j} I_{c j, p} \sum_{q=1}^{4} A_{q, l} I_{c l, q}\right),
\end{aligned}
$$

where $I_{c j, p}\left(I_{c j, 1}-I_{c j, 4}\right)$ and $I_{c l, q}\left(I_{c l, 1}-I_{c l, 4}\right)$ are given in the Appendix .

With the modal impedances due to $p_{\text {rad }}$ and $p_{\text {cav }}$ found, the dynamics Eq. (5) becomes a set of linear equations for the modal vibration amplitude, $V_{j}$,

$$
\left\{L_{j l}+Z_{+j l}-Z_{\mathrm{cav}, j l}\right\}\left\{V_{j}\right\}=-\left\{I_{j}\right\},
$$

where $\left\{V_{j}\right\},\left\{I_{j}\right\}$ are column vectors, and the modal coefficient of the incident wave, $I_{j}$, is defined as

$$
I_{j}=\int_{0}^{1} p_{i} \varphi_{j}(\xi) d \xi
$$

Equation (21) can be solved via the inversion of matrix. In the actual calculation, modal truncation is necessary. The plate modes are truncated to 25 . For instance, the subscripts of $j$ and $l$ in Eqs. (12), (16), and (20) range from 1 to 25, and the final impedance matrix $\left[Z_{j l}\right]$ is then of the size $25 \times 25$. For the duct acoustic mode $\psi_{n}$ in Eq. (14) and the cavity acoustic mode $\phi_{m, n}$ in Eq. (17), $m$ and $n$ are from 0 to 50. Numerical results show that the number of modes is normally enough as further increase in the number does not make significant difference for the purpose of this study.

The total sound pressure transmitted to the downstream is found by adding the incident wave, $p_{i}$, to the far-field radiation wave, $p_{+\mathrm{rad}}$, which can be found from Eq. (14) by taking only the plane wave mode $n=0$ for $x>L / 2$,

$$
p_{t}=\left.p_{+\operatorname{rad}}\right|_{n=0, x \rightarrow+\infty}+p_{i} .
$$

Similarly, the reflected wave is

$$
p_{r}=\frac{\left.p_{+\mathrm{rad}}\right|_{n=0, x \rightarrow-\infty}}{e^{i k_{0} x}},
$$

and the TL and the sound energy reflection coefficient $\beta$ are

$$
\mathrm{TL}=20 \log _{10} \frac{\left|p_{i}\right|}{\left|p_{t}\right|},
$$

and

$$
\beta=\frac{\left|p_{r}^{2}\right|}{\left|p_{i}^{2}\right|},
$$

respectively.

\section{SILENCER PERFORMANCE AND ITS OPTIMIZATION}

The main objective of this study is to develop a broadband wave reflector, which works preferably in the low frequency range. Thus, the performance of the plate silencer can be characterized by the widest stopband that can be achieved. Following the previous study (Huang, 2002), the stopband here is defined as the frequency range, $f \in\left[f_{1}, f_{2}\right]$, in which the TL is above a criterion value, $\mathrm{TL}_{c r}$, over the whole frequency band. As recommended in Huang (2004), the criterion value may be chosen as the peak TL for an expansion chamber whose cavity volume is three times the actual cavity volume in the silencer. For a silencer with two cavities of depth $h$ and length $L=5 h$, the value can be calculated and rounded up to $10 \mathrm{~dB}$. For the purpose of comparison with previous studies, the same configuration of cavity is adopted in the current study, and $\mathrm{TL}_{c r}=10 \mathrm{~dB}$ is chosen as the threshold level.

\section{A. Uniform clamped plates}

The performance of a side-branch plate silencer using uniform clamped plates is investigated. The default cavity shape is chosen as $L=5$ and $h_{c}=1$. For a given structure-toair mass ratio, $m$, the performance of the silencer is optimized by searching for the optimal bending stiffness $B_{\text {opt }}$ so that the bandwidth is maximum by varying other parameters such as the bending stiffness and, for the nonuniform plate described below, the thickness distribution. Since special emphasis is put on the low frequency noise, the cost function for the optimization is set as the ratio of the band limits, $f_{2} / f_{1}$, namely, the logarithmic bandwidth, instead of $f_{2}-f_{1}$. Results of the performance optimization are shown in Fig. 2.

Figure 2(a) compares the overall TL of the clamped plate silencer (solid line) with the drumlike silencer (dashed line). The mass ratios of the two models are both equal to 1 . For $B_{\text {opt }}=0.0698$, the lower and upper bandwidth limits of the plate silencer are $f_{1 c}=0.0445$ and $f_{2 c}=0.125$, respectively, and $f_{2 c} / f_{1 c}=2.81$. For the drumlike silencer with optimal tension $T_{\mathrm{opt}}=0.475$, the lower and upper bandwidth limits and bandwidth are $f_{1 d}=0.054, f_{2 d}=0.141$, and $f_{2 d} / f_{1 d}=2.6$, respectively. Clearly, with the same mass ratio $m=1$, the optimal performance of the clamped plate silencer is better than that of the drumlike silencer in terms of the lower band limit and the achievable bandwidth. Figures 

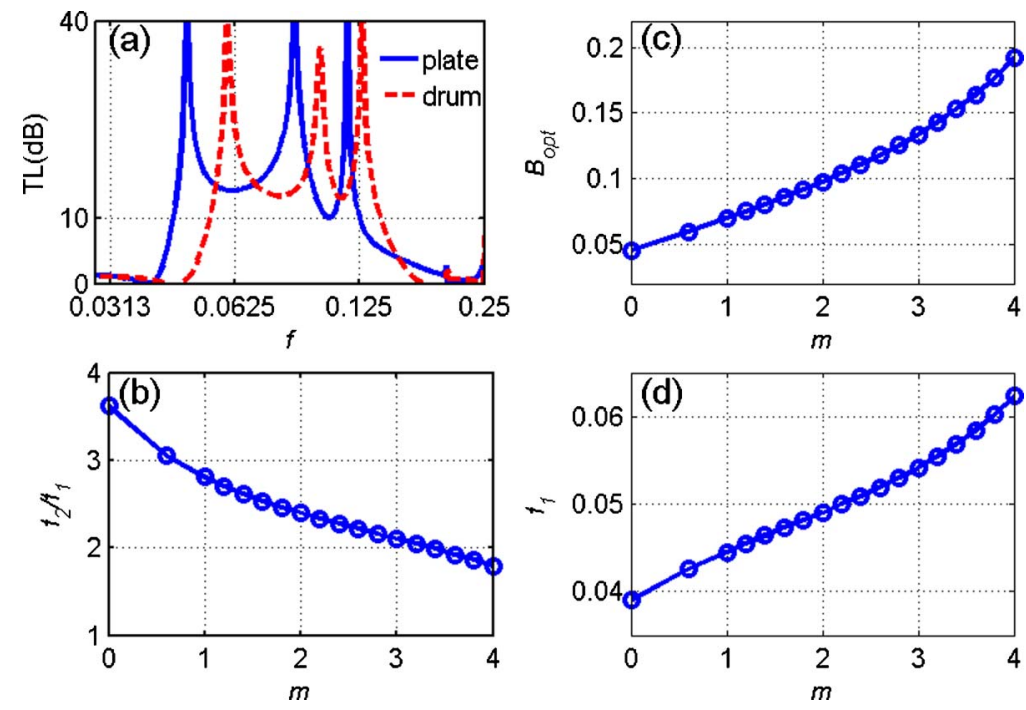

FIG. 2. (Color online) Performance optimization of the clamped plate silencer. (a) Comparison of the performance of the plate silencer (solid curve) with drumlike silencer (dashed curve) with the same cavity geometry of $L=5 h, h_{c}=h$, and the same mass ratio of $m=1$. (b) Bandwidth $f_{2} / f_{1}$. (c) Dimensionless optimal bending stiffness $B_{o p t}$. (d) Dimensionless lower band limit $f_{1}$.

2(b)-2(d) show the maximum bandwidth, the optimal bending stiffness, and the lower band limit as functions of $m$ for the clamped plate silencer. As shown in Fig. 2(b), when the plate mass increases, the maximum achievable bandwidth $f_{2} / f_{1}$ decreases gradually. When the mass ratio $m$ reaches 3 , the maximum bandwidth is 2.1 , which is still larger than one octave. Figure 2(c) suggests that the optimal bending stiffness increases with the plate mass, in much the same way as the simply supported plate silencer (Huang, 2006). However, since the clamped-clamped boundary conditions at the leading and trailing edges impose additional restrictions on the plate motion, the optimal bending stiffness required by the clamped-clamped plate is smaller than that of the corresponding simply supported plate of the same mass ratio. As shown in Fig. 2(c), the optimal bending stiffness for $m=1$ is $B_{\text {opt }}=0.0698$. For the simply supported plate, the corresponding $B_{\text {opt }}=0.1289$. When the plate mass increases, the lower band limit $f_{1}$ increases too, as shown in Fig. 2(d). Compared with the simply supported plate silencer, the lower band limit $f_{1}$ is a little higher when the clamped plate is adopted. This effect is undesirable for the plate silencer designed for lowfrequency noise control. Nevertheless, the lower band limits are still within the low frequency region. For example, when $m=3, f_{1}=0.054$. For a duct with a height of $20 \mathrm{~cm}$, the dimensional lower band limit is $f_{1}^{*}=92 \mathrm{~Hz}$.

Generally speaking, the performance of the clamped plate silencer using uniform plates is good, but it is not as attractive as that of the simply supported plate silencer. As presented in Huang (2006), when $m=1$, a simply supported plate silencer can achieve a stopband of $f_{2} / f_{1}=4.25$ with $f_{1}$ $=0.0353$. For the current model the two corresponding indi- cators are 2.81 and 0.0445 . The performance degradation of the clamped plate silencer may be attributed to the clamped conditions applied to the leading and trailing edges of the plate. Such a boundary condition brings additional restrictions to portions of the plate near the ends, which in turn prevents the plate from vibrating and radiating reflection sound effectively. To further increase the stopband and reduce the lower band limit $f_{1}$, the use of a nonuniform plate with softer ends is explored. In the next subsection, the optimal bending stiffness distribution along the length of the plate is investigated, and the optimal TL is analyzed and compared with that of the simply supported plate silencer.

\section{B. Nonuniform plate}

The so-called optimization of nonuniform distribution $B(x)$ is realized by choosing one shape from a set of possibilities that can yield the widest stopband. When constructing $B(x)$, the following two factors are taken into considerations. First, the purpose of introducing the nonuniform plate is to release the structural restrictions near the clamped ends; therefore, the bending stiffness near the two ends should be smaller than that in the middle. Second, the lower vibration modes (especially the first two) of the plate are dominant in the reflection of sound. Based on the above two considerations, it is appropriate to construct $B(x)$ as

$$
B(x)=B_{0} d(x),
$$

where $B_{0}$ is a constant ratio to be determined, and $d(x)$ is the shape function of the bending stiffness. One such distribution is shown in Fig. 3(a) and is described below,

$d(\xi)= \begin{cases}h_{1}+\frac{\arctan \left(\alpha_{1} \xi_{1}\right)+\arctan \left[\alpha_{1}\left(\xi-\xi_{1}\right)\right]}{\arctan \left(\alpha_{1} \xi_{1}\right)+\arctan \left[\alpha_{1}\left(\xi_{0}-\xi_{1}\right)\right]}\left(1-h_{1}\right), & 0<\xi \leq \xi_{0}, \\ h_{2}+\frac{\arctan \left[\alpha_{2}\left(1-\xi_{2}\right)\right]-\arctan \left[\alpha_{2}\left(\xi-\xi_{2}\right)\right]}{\arctan \left[\alpha_{2}\left(1-\xi_{2}\right)\right]-\arctan \left[\alpha_{2}\left(\xi_{0}-\xi_{2}\right)\right]}\left(1-h_{2}\right), & \xi_{0}<\xi<1,\end{cases}$ 

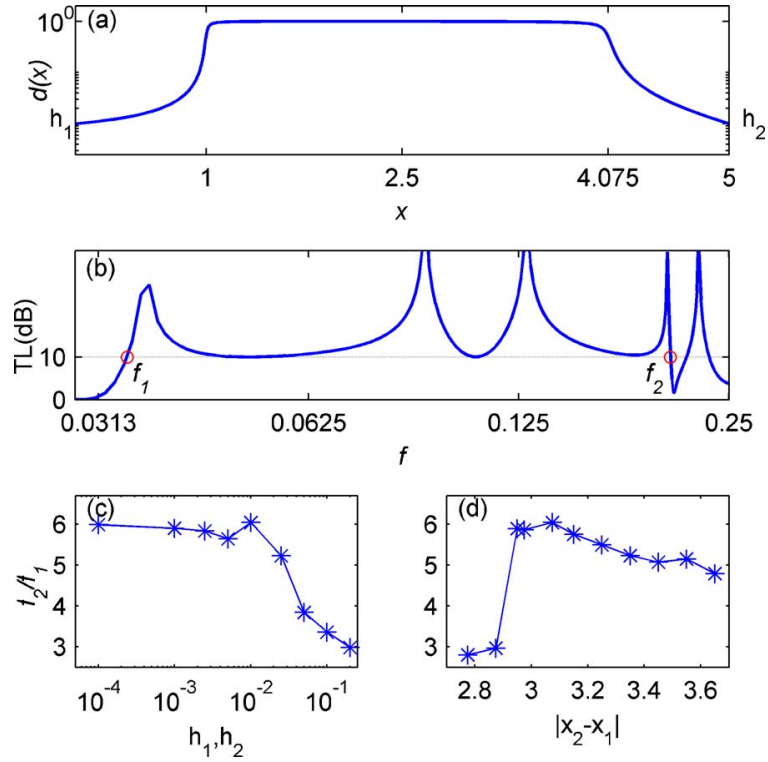

FIG. 3. (Color online) Optimization of the nonuniform plate. (a) Optimal distribution of the bending stiffness along the length of the plate. (b) TL. (c) Bandwidth $f_{2} / f_{1}$ with respect to the relative bending stiffness at the two thin ends. (d) Bandwidth $f_{2} / f_{1}$ with respect to the length of the thick part of the plate. The cavity geometry is $L=5 h, h_{c}=h$; average mass ratio $\bar{m}=1$.

where $\xi=(x / L+1 / 2)$, as defined in Eq. (6). There are seven control parameters in the above-defined function $d(\xi)$, namely, $\alpha_{1}, \alpha_{2}, h_{1}, h_{2}, \xi_{0}, \xi_{1}$, and $\xi_{2}$, where $\alpha_{1}, \alpha_{2}$ control the slope of the resulting curve; $h_{1}, h_{2}$ control the heights of the curve at the two ends; and $\xi_{0}, \xi_{1}$, and $\xi_{2}$ control the horizontal positions of the two segments. Since $B(x)$ plays a more important role in the dynamics of the plate than $m(x)$ does, the distribution of mass ratio, $m(x)$, is simply taken as

$$
m(x)=m_{0} \sqrt[3]{d(x)} .
$$

The cavity geometry is still assumed to be $h_{c}=1$ and $L=5$. The ranges of the seven controlling parameters are $h_{1}, h_{2}$ $\in[0.0001,0.2], \quad \alpha_{1}, \quad \alpha_{2} \in[1,1500], \quad \xi_{0} \in[0.3,0.6], \quad \xi_{1}$ $\in[0.05,0.25]$, and $\xi_{2} \in[0.75,0.95]$. During the optimization, the seven parameters vary step by step within the ranges given above. The TL is calculated for each given set of parameters, and the set of parameters that results in the widest stopband is chosen as the optimal one. The corresponding $B(x)$ is then the optimal distribution of the bending stiffness. In order to facilitate the comparison of the TL spectrum for each $B(x)$, the total mass along the plate is kept as a constant during the optimization,

$$
\int_{0}^{L} m(x) d x=\bar{m} L
$$

where $\bar{m}=1$ is chosen in the example given. For the nonuniform plate, $\bar{m}$ is the average mass ratio along the length of the plate. The maximum stopband $f \in[0.0343,0.2062]$ is found with the following parameters:

$$
\begin{aligned}
& \xi_{0}=0.35, \quad \xi_{1}=0.2, \quad \xi_{2}=0.815, \quad \alpha_{1}=300, \\
& \alpha_{2}=100, \quad h_{1}=h_{2}=0.01
\end{aligned}
$$
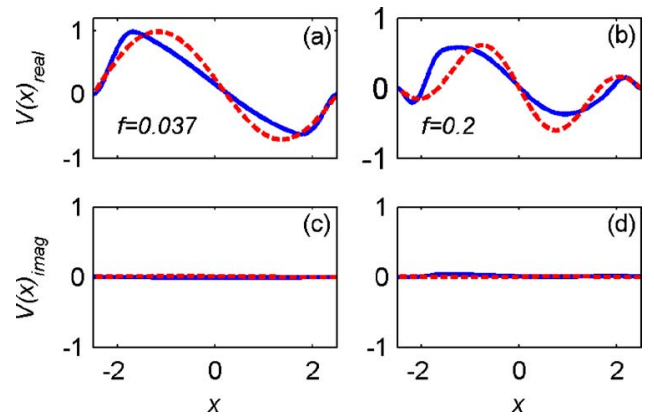

FIG. 4. (Color online) Comparison of the vibration velocity distribution $V(x)$ between the clamped nonuniform plate (solid lines) and the simply supported plate (dashed lines). (a) $f=0.037$, real parts. (b) $f=0.2$, real parts. (c) $f=0.037$, imaginary parts. (d) $f=0.2$, imaginary parts.

The constant $B_{0}$ is determined as 0.4935 . The results of the optimization are shown in Fig. 3.

Figure 3(a) gives the optimal distribution of the bending stiffness along the length of the plate. Parameters $h_{1}$ and $h_{2}$ represent the relative bending stiffness at the two edges, and both of them are equal to 0.01 in this case. As expected, the two ends of the plate are much softer than the portion in the middle and the additional restriction due to the clamped boundary conditions is thus released. The length of the thick portion in the middle can be roughly characterized as $\mid \xi_{2}$ $-\xi_{1} \mid$, which is 0.615 in this case. Suppose the plate material is homogeneous. Such a distribution can be realized approximately by making the thickness of the plate into the shape of $\sqrt[3]{d(x)}$. Figure $3(\mathrm{~b})$ shows the overall TL using a logarithmic scale. The stopband begins from $f_{1}=0.0343$ and ends at $f_{2}$ $=0.2063$, the bandwidth being $f_{2} / f_{1}=6.02$. Four peaks can be observed in the stopband. Figure 3(c) shows the maximum bandwidth as a function of $h_{1}$ and $h_{2}$. Here, values of other parameters given in Eq. (31) are used. A softer end can help reduce the restriction due to the clamp condition. However, it does not follow that the softer the two ends are, the better the performance is. As shown in Fig. 3(c), when $h_{1}$, $h_{2}=0.01$, the bandwidth reaches the maximum of 6.02. Further decrease in $h_{1}$ and $h_{2}$ does not make any noticeable improvement of the bandwidth. Figure 3(d) shows the bandwidth $f_{2} / f_{1}$ as a function of the length of the thick portion, $\left|\xi_{2}-\xi_{1}\right|$, while other parameters are kept as the optimal values given in Eq. (31). As $\left|\xi_{2}-\xi_{1}\right|$ decreases from the optimal point, the performance drops and approaches the behavior of a uniform plate.

Figure 4 compares the vibration velocity distribution $V(x)$ between the clamped nonuniform plate (solid lines) and a simply supported plate (dashed lines) at $f=0.037$ (the first peak) and $f=0.2$ (near the fourth peak). The real parts are shown in Figs. 4(a) and 4(b). Since the plate vibrations are almost in-phase over the entire length, the imaginary parts of $V(x)$ shown in Figs. 4(c) and 4(d) are almost zero. As expected, the clamped plate with softer ends responds in a similar way to the simply supported plate, but the peaks and troughs are much closer to the clamped ends. The effect of this difference is as follows. The plate vibration is dominated by a mode shape that is close to a dipolelike pattern of sound radiation. Much of the sound radiated by the leading edge portion of the plate with $V(x)>0$, cf. Fig. 4(a), is cancelled 

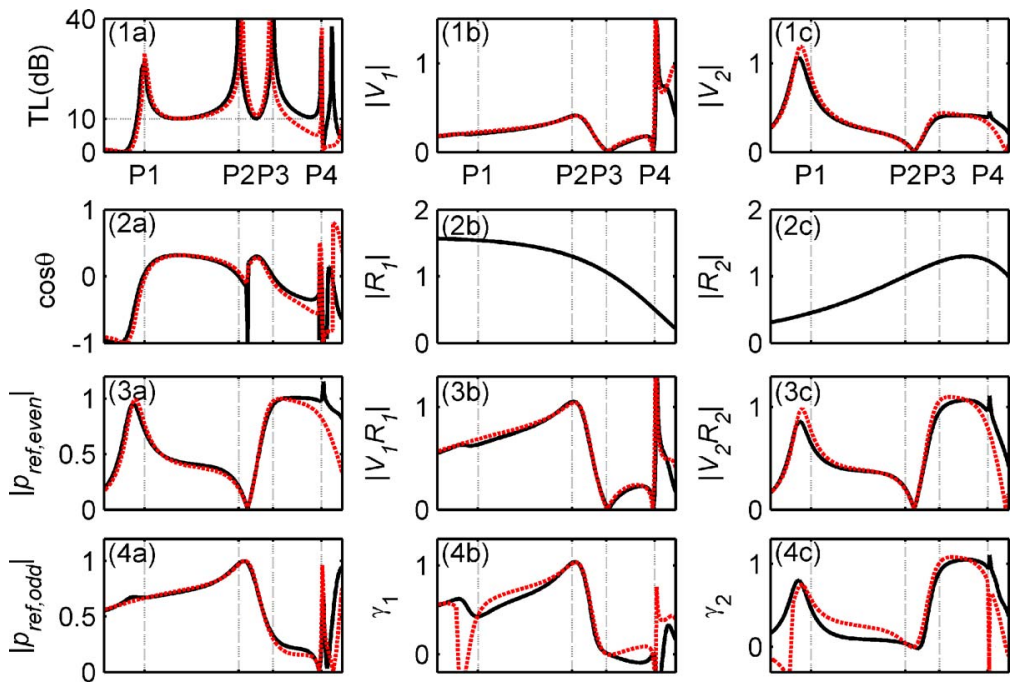

FIG. 5. (Color online) Comparison of the clamped nonuniform plate with the simply supported uniform plate (solid lines: nonuniform plate; dashed lines: simply supported). The cavity geometry is $L=5 h, h_{c}=h$, and the average mass ratio $\bar{m}=1$. (1a) TL. (2a) Odd-even modal interference index. (3a) Sound reflected by the even modes. (4a) Sound reflected by the odd modes. The second and third columns compare the modal amplitude, $\left|V_{j}\right|$, the modal reflection coefficient, $\left|R_{j}\right|$, the single mode reflection, $\left|V_{j} R_{j}\right|$, and the modal contribution $\gamma_{j}$ of the two plate silencers. The four peak positions are identified in all the subfigures. by that from the trailing edge portion where $V(x)<0$. When $f \rightarrow 0$, this cancellation would be rather complete since the plate length is negligible compared with the wavelength of sound in air. The length between the peaks and troughs in Figs. 4(a) and 4(b) thus determines the radiation efficiency of the plate for a finite frequency. In other words, a clamped plate with much of the length behaving like a rigid plate has a higher capability to reflect sound, in a dipolelike mode that is not prohibited by the cavity stiffness.

The performance of the nonuniform clamped plate silencer with that of the simply supported plate is compared and shown in Fig. 5. The cavity geometry and the mass ratio of the two silencers are the same, namely, $L=5, h_{c}=1$, and $\bar{m}=1$. The optimal bending stiffness $B_{\text {opt }}$ of the simply supported plate is 0.129 (Huang, 2006). The results for the nonuniform plate silencer are shown in solid lines and those of the simply supported plate silencer in dashed lines. The first column compares the TL spectrum, odd-even modal interference index, and reflection sound due to odd and even modes. P1 through P4 identify the four peak positions. As shown in Fig. 5(1a), the performance of the clamped nonuniform plate silencer can be even better than that of the model using simply supported uniform plates. The difference mainly comes from the relatively high frequency region, namely, from $\mathrm{P} 3$ to $\mathrm{P} 4$. From $\mathrm{P} 1$ to $\mathrm{P} 3$, the reflection sound and odd-even modal interference index of the two silencers are almost identical, which results in a similar TL for the two silencers. The second and third columns compare the modal amplitude, $\left|V_{j}\right|$, modal reflection coefficient, $\left|R_{j}\right|$, the single mode reflection, $\left|V_{j} R_{j}\right|$, and the modal contribution, $\gamma_{j}$, of the first two modes. Here, $R_{j}$ is the complex amplitude of the reflected sound by the induced vibration of the $j$ th mode with unit amplitude. To facilitate the comparison, the vibration mode of a simply supported plate, $\sin (j \pi \xi)$, is used for both silencers. Hence, the expression for $R_{j}$ given in Huang (2002) still applies. The $\gamma_{j}$ represents the contribution of each single mode, denoted as $V_{j} R_{j}$, towards the total reflected sound $p_{r}$. When a nonuniform plate is used, the local peaks of both $\gamma_{1}$ and $\gamma_{2}$ near the first peak point P1 are pushed to lower frequency, which results in a smaller lower bound $f_{1}$. The high TL between the third peak point $\mathrm{P} 3$ and the upper bound $f_{2}$ are mainly due to the increased $\left|V_{2}\right|,\left|V_{2} R_{2}\right|$ and $\gamma_{2}$, as shown in Figs. 5(1c), 5(3c), and 5(4c). Therefore, compared with the uniform plate, a properly designed nonuniform plate can greatly enhance the performance of the clamped plate silencer.

\section{EXPERIMENTAL VALIDATION}

The main objective of the experimental study is to validate the basic theoretical model established. The TL of a prototyped clamped plate silencer was measured and compared with the theoretical prediction. In doing so, a number of issues that might complicate the task are put aside at this stage. One of these issues is the modeling of the dynamics of a plate of nonuniform thickness and the actual preparation of such thickness distribution for a thin plate. The following experiment is therefore limited to the test of a uniform plate. The issue of structural damping is another difficult one, but it cannot be avoided altogether. An attempt is made to simulate the damping effect towards the end of this section.

\section{A. Experimental rig}

The TL of the plate silencer was measured by the fourmicrophone, two-load method similar to the one described by Munjal and Doige (1990), as shown in Fig. 6. The incident noise is simulated by a loudspeaker connected to the duct through a contraction cone. The output signal from the DA converter (NI. PCI-M10-16E-1) was passed to the loudspeaker via a power amplifier (B\&K's LAB Gruppen 300). Two pairs of 1/2-in., phase-matched microphones (B\&K type 4187), labelled as M1 through M4, were used together with conditioning amplifier (B\&K Nexus 2691). The separation distances between the microphones and their exact locations are indicated in Fig. 6. Signals from the four microphones were acquired via the AD converter (NI. PCI-4452). Both $\mathrm{A} / \mathrm{D}$ and D/A processes were controlled by an NI Labview program and the test was run by a loop of discrete frequencies from 40 to $800 \mathrm{~Hz}$ with an increment of $10 \mathrm{~Hz}$. Two linearly independent experiments with different downstream loading conditions were conducted to determine the TL of the tested silencer. In the current study, the first downstream 


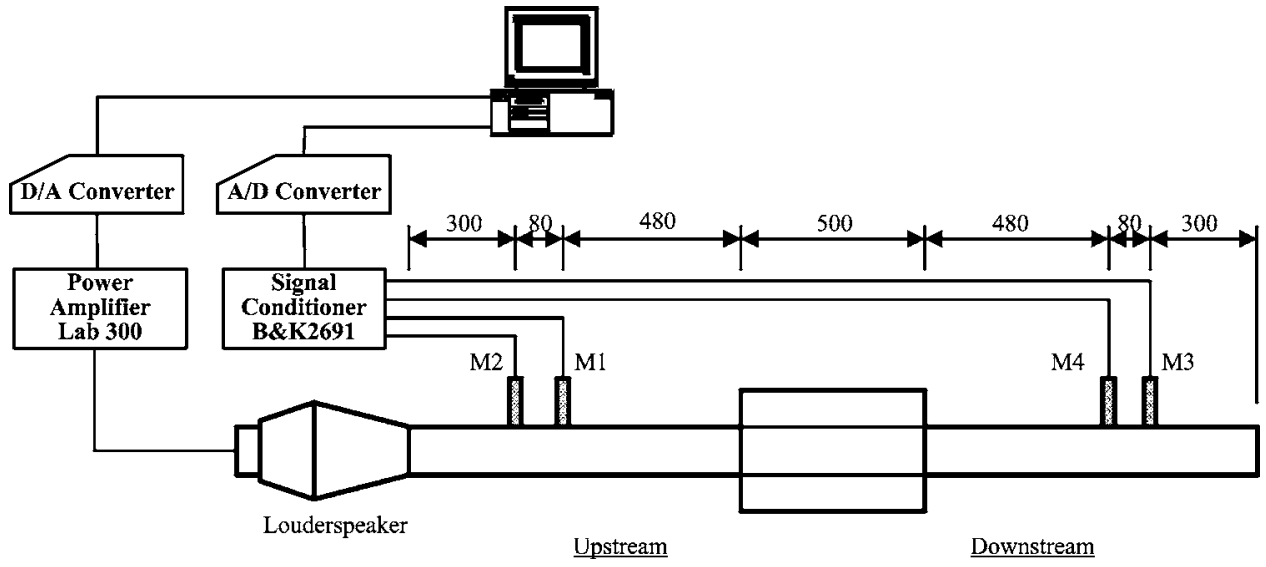

$\underline{\text { Upstream }}$
FIG. 6. The four-microphone, twoload measurement system (dimensions in $\mathrm{mm})$. boundary condition was a simple "rigid ending." The other one was "absorption ending," which was implemented by connecting the downstream end of the duct to an absorptive chamber.

The duct wall was made of 15-mm-thick acrylic and was considered to be acoustically rigid. The cross-section of the duct was $100 \times 100 \mathrm{~mm}^{2}$. The two cavities also had a cross section of $100 \times 100 \mathrm{~mm}^{2}$ and the length was $500 \mathrm{~mm}$. With the geometry given, the first cut-on frequency in the rigid duct is about $1700 \mathrm{~Hz}$, which is much higher than the upper limit of the measurement frequency range. Two pieces of balsawood plates were installed flush with the duct wall. The plates were $517 \mathrm{~mm}$ long, $102 \mathrm{~mm}$ wide, and $2 \mathrm{~mm}$ thick. The leading and trailing edges of the plates were clamped, and the final effective length of the plates was $500 \mathrm{~mm}$. The two lateral edges of the plates were inserted into a thin gap between the two constituent plates of the cavity walls. The clearance between the lateral edges of the plates and the cavity walls was less than $0.5 \mathrm{~mm}$. Such a configuration was adopted to make sure that the plates could vibrate freely and simulate the two-dimensional behavior of a beam while the leakage of noise from the main duct to the cavities via the gap was minimized.

The physical and mechanical properties of balsawood vary greatly depending on the origin and growth conditions. According to Chart 1 given in Ashby (2005), the density of balsawood ranges from 100 to $220 \mathrm{~kg} / \mathrm{m}^{3}$, and the Young's modulus from 2 to $5 \mathrm{GPa}$. Since the bending stiffness of the plate is one of the most crucial design factors, a three-point bending test was conducted to determine it experimentally. Figure 7(a) shows the schematic of the three-point bending test. The sample plate was of the size $90 \times 32 \times 2 \mathrm{~mm}^{3}$, and the support span $L$ was $50 \mathrm{~mm}$. An axial static testing machine was used to apply the load gradually. The relationship between the applied load and the extension at the middle point of the plate is shown in Fig. 7(b). The response of the plate was rather linear as the extension increases from 0 to $1.5 \mathrm{~mm}$. Hence, the measured data within this region were used to find the Young's modulus. For a simply supported beam given in this test, the extension at the middle point and the applied load should satisfy the relationship

$$
y=\frac{F L^{3}}{48 E I},
$$

where $y$ is the extension, $F$ is the load applied, $E$ is the Young's modulus, and $I$ is the second moment of inertia. According to Fig. 7(b), the Young's modulus $E$ can be estimated as $2.6 \mathrm{GPa}$. The measured density of the balsa wood plate was $208 \mathrm{~kg} / \mathrm{m}^{3}$. Therefore, the dimensionless bending stiffness and mass ratio of the plate in the silencer can be calculated by the scheme described in Eq. (2), and the final results are $B=0.0123$ and $m=3.4$.

Before measuring the TL of the plate silencer, the TL of the whole measurement system without the balsawood plates was first measured. This measurement serves two purposes. One is to calibrate the measurement system, and the other is to check the damping level of the duct and the cavity. The TL spectrum, the energy absorption coefficient $\alpha$, and the energy reflection coefficient $\beta$ were measured and compared with the theoretical predictions in Fig. 8. With the plate absent, the plate silencer became a rectangular expansion chamber. The theoretical results were obtained by assuming both the mass ratio and bending stiffness to be zero. Figure 8(a) indicates that the measured TL (the line with open circles) compares with the theoretical solution well on the whole, except

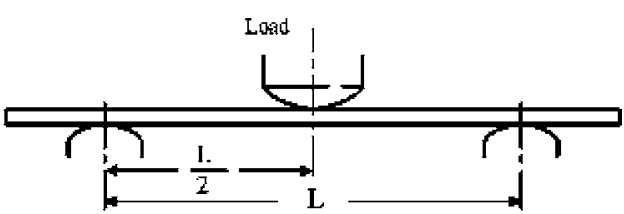

(a)

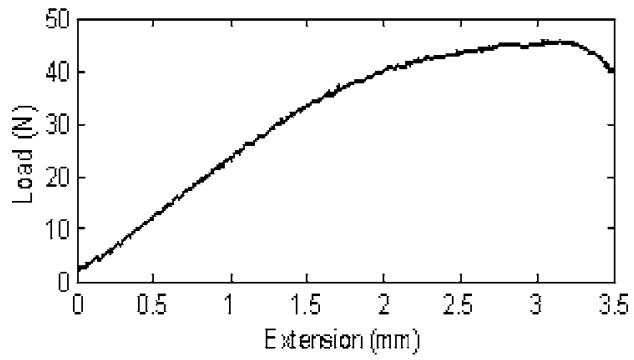

(b)

FIG. 7. Three-point bending test of the balsawood plate. (a) Schematic of the bending test. (b) Load versus extension at the middle point. 

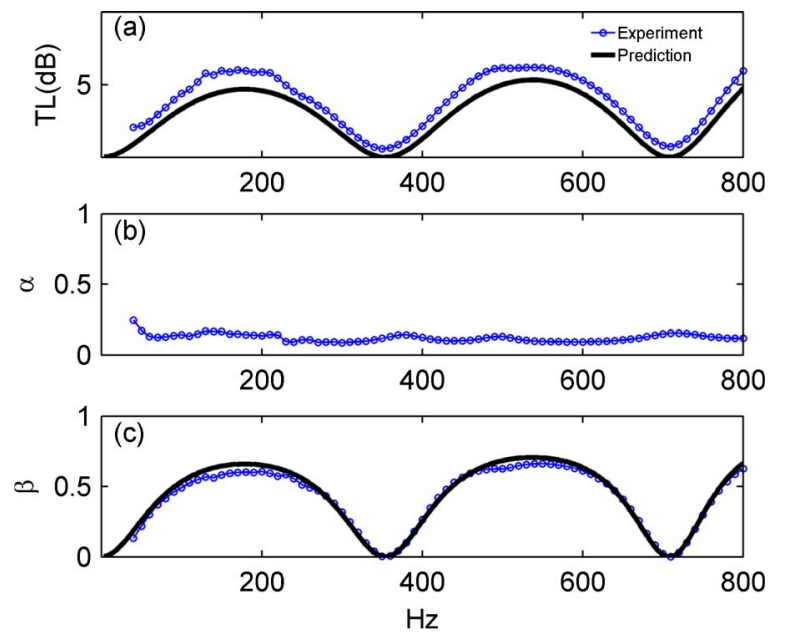

FIG. 8. (Color online) Comparison of the measured data (with open circles) with theoretical solution (without open circles) for the expansion chamber. (a) TL. (b) The absorption coefficient $\alpha$. (c) The reflection coefficient $\beta$.

that the measured $\mathrm{TL}$ is $0.5 \mathrm{~dB}$ higher than the prediction. The discrepancy is mainly due to the energy dissipation mechanisms, which are necessarily present in the rig but excluded in the theory. As shown in Fig. 8(b), the overall energy absorption coefficient of the expansion chamber is around 0.1 . The possible damping mechanism includes the cavity damping, wall vibration, etc.

\section{B. Results analysis}

The TL and the corresponding energy reflection coefficient $\beta$ of the tested plate silencer were first calculated using the parameters found in the last section $(B=0.0123, m=3.4)$. The predicted results are shown in Figs. 9(a) and 9(c) in solid curves. Since the damping effect was not considered in the theoretical model, the predicted absorption coefficient was zero. The frequency positions of the six predicted peaks in Fig. 9(a) are 54, 109, 224, 306, 415, and 727 Hz. Because the bending stiffness of the plate used is much smaller than the optimal one [see Fig. 2(c)], the TL between the first and
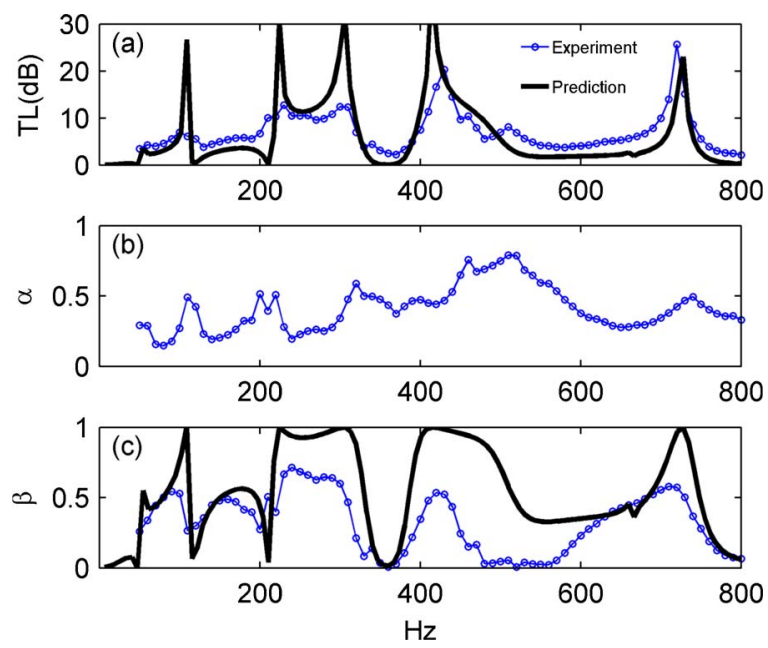

FIG. 9. (Color online) Comparison of experimental data (solid curves with open circles) with theoretical solution (solid curves without open circles). (a) TL. (b) The absorption coefficient $\alpha$. (c) The reflection coefficient $\beta$. The dimensionless bending stiffness $B=0.0123$, the mass ratio $m=3.4$. second peaks is low, and the widest stopband begins from $218 \mathrm{~Hz}$ and ends at $318 \mathrm{~Hz}$, consisting of the third and fourth peaks, but it suffices to serve the objective of model validation. Note that the balsawood used here is relatively heavy and soft in the whole range of balsawood available. For better performance of the plate silencer, a lighter and stiffer balsawood may be used.

Figure 9(a) compares the measured TL (solid curves with open circles) with the predicted one. Evidently, the spectral peaks and the overall spectral shapes of the measured TL match those of the theoretical results. This means that the basic theoretical model is correct for the experimental rig. However, the predicted TL curve has sharp peaks while that from the experiment is quite smooth. This, again, implies significant energy dissipation mechanisms in the experimental rig, which are excluded in the theoretical model. As shown in Fig. 9(b), the measured absorption coefficient $\alpha$ ranges from 0.15 to 0.5 in the low frequency region (say, below $400 \mathrm{~Hz}$ ) and from 0.3 to 0.8 in the relative high frequency region (above $400 \mathrm{~Hz}$ ). Note that the sound absorption coefficient is only around 0.1 when the balsawood plates are absent. It is reasonable to assume that the main energy dissipation mechanism in the plate silencer is the structural damping in the plate. Figure 9(c) compares the energy reflection coefficient $\beta$. Obviously, the level of the measured reflection coefficient is lower than prediction. One explanation for this phenomenon is that the structural damping reduces the vibration level of the balsawood plates, which in turn results in a lower level of sound reflection. On the other hand, the existence of sound absorption also lowers the chances of the incident noise being reflected. Nevertheless, comparison between Figs. 9(b) and 9(c) shows that the mechanism of sound reflection still dominates over the sound absorption in the low frequency range. To summarize, the experimental results are in agreement with the theoretical prediction, but the damping effect, which is excluded previously, should be considered in the theoretical model to better represent the real physics behind the plate silencer.

The damping effect is now considered in the theoretical model by the Rayleigh damping model, and the coupled dynamics Eq. (3) becomes, for harmonic vibration of time dependence $e^{i \omega t}$,

$$
\frac{\partial^{2}}{\partial x^{2}}\left(B(x) \frac{\partial^{2} \eta}{\partial x^{2}}\right)\left(1+i \sigma_{s}\right)+m(x) \frac{\partial^{2} \eta}{\partial t^{2}}\left(1-i \sigma_{m}\right)+\left(p_{i}+\Delta p\right)=0,
$$

where $\sigma_{s}$ and $\sigma_{m}$ are, respectively, the stiffness and mass damping coefficients. Two cases with different damping coefficients are investigated. In the first case, $\sigma_{m}=0.15$ and $\sigma_{s}=0$. The TL, absorption coefficient, and reflection coefficient are shown in Figs. 10(1a)-10(1c), respectively. It can be observed that the experimental data are in good agreement with the theoretical results when the frequency is below $360 \mathrm{~Hz}$. However, the absorption coefficient is underestimated in high frequency. In the other case, the damping coefficients are chosen as $\sigma_{m}=\sigma_{s}=0.05$, and the results are shown in Figs. 10(2a)-10(2c). With such a combination of $\sigma_{m}$ and $\sigma_{s}$, the measured TL, absorption 

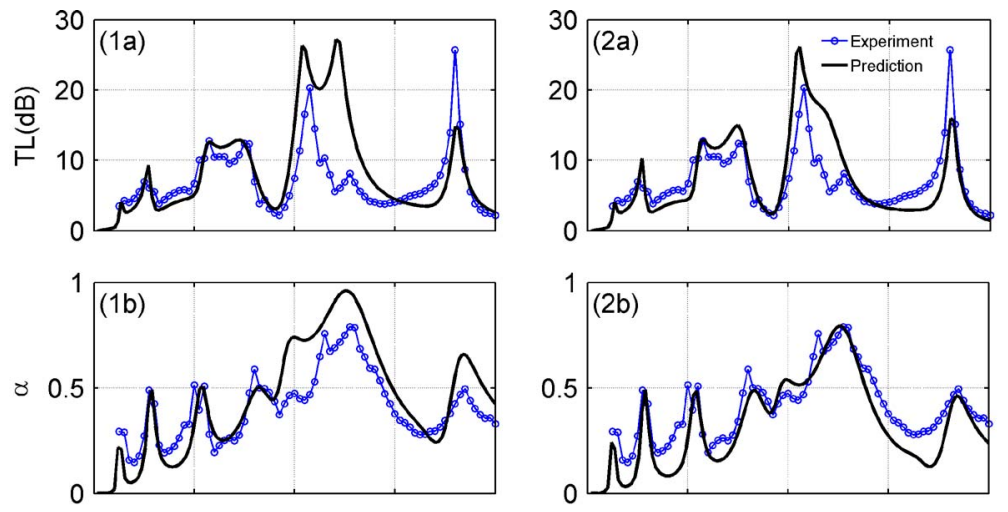

FIG. 10. (Color online) Modeling of plate damping mechanisms with two set of damping factors. (1a) and (1b), and (1c) show the results with $\sigma_{m}=0.15, \sigma_{s}=0$. (2a), (2b), and (2c) are the results with $\sigma_{m}=0.05, \sigma_{s}$ $=0.05$.
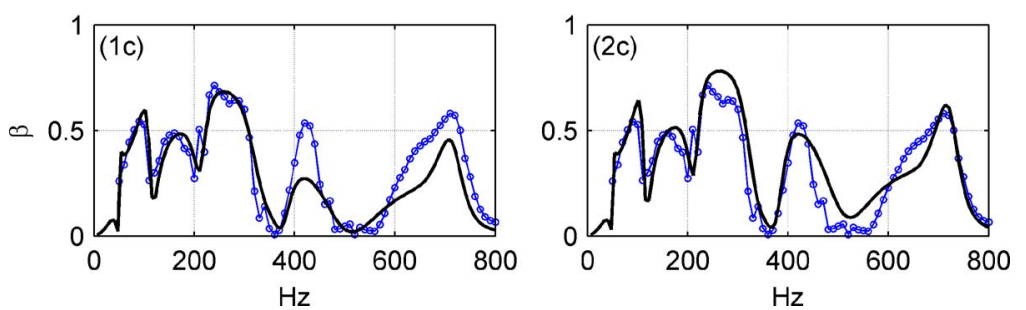

coefficient, and reflection coefficient are all in agreement with the theoretical results on the whole. But in the frequency range of 240 to $300 \mathrm{~Hz}$, the reflection coefficient is overestimated slightly. Since low frequency noise is of major interest in this study, the first damping model may be more suitable for design purposes.

\section{CONCLUSIONS}

It is easy to realize a side-branch plate silencer with the plate clamped at both the leading and trailing edges in practice. Theoretical study reveals that such a plate silencer can function effectively as a low frequency wave reflector over a broad frequency band. When uniform plates are used, a stopband above one octave can be achieved if the plate-to-air mass ratio is less than 3.5. The performance of the clamped plate silencer can be further enhanced by using plates with softer ends. Optimization study shows that, with a proper distribution of the bending stiffness along the plate, the widest stopband of the clamped plate silencer may be up to $f_{2} / f_{1}=6.0$ for a mass ratio of $m=1$, which is even wider than that of a simply supported plate silencer.

When excited by the incident wave, the clamped nonuniform plate responds in a similar way to the simply supported plate, but the displacement peaks and troughs are much closer to the clamped ends. The extended length between the peaks and troughs makes the nonuniform plate

more capable of reflecting sound in a dipolelike mode, which is not prohibited by the cavity. Comparison of the modal reflections between the clamped nonuniform plate and the simply supported plate shows that the second in vacuo plate mode contributes most to the expansion of the stopband of the nonuniform plate silencer, especially in the high frequency region, which, to some extent, conforms to the above explanation.

Experimental study demonstrated that the basic model of the clamped plate silencer is correct. The spectral peaks and the shapes of the measured TL are in agreement with those calculated theoretically. In the low frequency range, say, below $400 \mathrm{~Hz}$, the sound reflection still dominates over the sound energy dissipation. Significant sound absorption exists in the plate silencer, especially at high frequencies. A Rayleigh damping model is adopted to represent the plate damping in the testing rig. Results show that with a proper combination of mass and stiffness damping coefficients, the simulated damping effect is in close agreement with the experimental data.

\section{ACKNOWLEDGMENTS}

The project is supported by a research grant from the Hong Kong SAR government (PolyU 5298/03E). The first two authors thank the Hong Kong Polytechnic University for the research studentships.

\section{APPENDIX: RESULTS OF $I_{p, q}, I_{c j, p}$, AND $I_{c l, q}$}

When $j \neq l$, 9 out of the 16 components of $I_{p, q}\left(I_{1,1} \sim I_{4,4}\right)$ are given below:

$$
\begin{aligned}
& I_{1,1}=\frac{2 i k_{n} L\left(1-e^{\left.\beta_{j}+\beta_{l}\right)}\right.}{\left[\beta_{j}^{2}+\left(k_{n} L\right)^{2}\right]\left(\beta_{j}+\beta_{l}\right)}-\frac{e^{\beta_{l}-i k_{n} L}-1}{\left(\beta_{j}+i k_{n} L\right)\left(\beta_{l}-i k_{n} L\right)}+\frac{e^{\beta_{j}+\beta_{l}}-e^{\beta_{j}-i k_{n} L}}{\left(\beta_{j}-i k_{n} L\right)\left(\beta_{l}+i k_{n} L\right)}, \\
& I_{1,3}=\frac{\beta_{j}\left(e^{\beta_{l}-i k_{n} L}-1\right)}{\left(\beta_{l}-i k_{n} L\right)\left[\beta_{j}^{2}-\left(k_{n} L\right)^{2}\right]}+\frac{2 i k_{n} L\left[\left(\beta_{l} \sin \beta_{j}-\beta_{j} \cos \beta_{j}\right) e^{\beta_{l}}+\beta_{j}\right]}{\left(\beta_{j}^{2}+\beta_{l}^{2}\right)\left[\beta_{j}^{2}-\left(k_{n} L\right)^{2}\right]}-\frac{\left(e^{\beta_{l}}-e^{-i k_{n} L}\right)\left(i k_{n} L \sin \beta_{j}+\beta_{j} \cos \beta_{j}\right)}{\left(\beta_{l}+i k_{n} L\right)\left[\beta_{j}^{2}-\left(k_{n} L\right)^{2}\right]},
\end{aligned}
$$




$$
\begin{aligned}
& I_{1,4}=\frac{2 i k_{n} L}{\beta_{j}^{2}-\left(k_{n} L\right)^{2}}\left[\frac{\left(\beta_{j} \sin \beta_{j}+\beta_{l} \cos \beta_{j}\right) e^{\beta_{l}}-\beta_{l}}{\beta_{j}^{2}+\beta_{l}^{2}}-\frac{e^{\beta_{l}-i k_{n} L}-1}{2\left(\beta_{l}-i k_{n} L\right)}\right]+\frac{\left(e^{\beta_{l}}-e^{-i k_{n} L}\right)\left(\beta_{j} \sin \beta_{j}-i k_{n} L \cos \beta_{j}\right)}{\left(\beta_{l}+i k_{n} L\right)\left[\beta_{j}^{2}-\left(k_{n} L\right)^{2}\right]}, \\
& I_{3,1}=\frac{2 i k_{n} L\left[\left(\beta_{l} \cos \beta_{l}-\beta_{j} \sin \beta_{l}\right) e^{\beta_{j}}-\beta_{l}\right]}{\left(\beta_{j}^{2}+\beta_{l}^{2}\right)\left[\beta_{j}^{2}+\left(k_{n} L\right)^{2}\right]}+\frac{\left(i k_{n} L \sin \beta_{l}-\beta_{l} \cos \beta_{l}\right) e^{\beta_{j}}+\beta_{l} e^{\beta_{j}-i k_{n} L}}{\left(\beta_{j}-i k_{n} L\right)\left[\beta_{l}^{2}-\left(k_{n} L\right)^{2}\right]}-\frac{\beta_{l}-\left(i k_{n} L \sin \beta_{l}+\beta_{l} \cos \beta_{l}\right) e^{-i k_{n} L}}{\left(\beta_{j}+i k_{n} L\right)\left[\beta_{l}^{2}-\left(k_{n} L\right)^{2}\right]}, \\
& I_{3,3}=\frac{\beta_{j}\left[\beta_{l}-\left(i k_{n} L \sin \beta_{l}+\beta_{l} \cos \beta_{l}\right) e^{-i k_{n} L}\right]}{\left[\beta_{j}^{2}-\left(k_{n} L\right)^{2}\right]\left[\beta_{l}^{2}-\left(k_{n} L\right)^{2}\right]}+\frac{i k_{n} L}{\beta_{j}^{2}-\left(k_{n} L\right)^{2}}\left[\frac{\sin \left(\beta_{j}-\beta_{l}\right)}{\beta_{j}-\beta_{l}}-\frac{\sin \left(\beta_{j}+\beta_{l}\right)}{\beta_{j}+\beta_{l}}\right] \\
& -\frac{\left(i k_{n} L \sin \beta_{j}+\beta_{j} \cos \beta_{j}\right)\left[\left(i k_{n} L \sin \beta_{l}-\beta_{l} \cos \beta_{l}\right)+\beta_{l} e^{-i k_{n} L}\right]}{\left[\beta_{j}^{2}-\left(k_{n} L\right)^{2}\right]\left[\beta_{l}^{2}-\left(k_{n} L\right)^{2}\right]}, \\
& I_{3,4}=\frac{i k_{n} L\left[\left(i k_{n} L \sin \beta_{l}+\beta_{l} \cos \beta_{l}\right) e^{-i k_{n} L}-\beta_{l}\right]}{\left[\beta_{j}^{2}-\left(k_{n} L\right)^{2}\right]\left[\beta_{l}^{2}-\left(k_{n} L\right)^{2}\right]}+\frac{i k_{n} L}{\beta_{j}^{2}-\left(k_{n} L\right)^{2}}\left[\frac{1-\cos \left(\beta_{j}+\beta_{l}\right)}{\beta_{j}+\beta_{l}}-\frac{1-\cos \left(\beta_{j}-\beta_{l}\right)}{\beta_{j}-\beta_{l}}\right] \\
& +\frac{\left(\beta_{j} \sin \beta_{j}-i k_{n} L \cos \beta_{j}\right)\left[\left(i k_{n} L \sin \beta_{l}-\beta_{l} \cos \beta_{l}\right)+\beta_{l} e^{-i k_{n} L}\right]}{\left[\beta_{j}^{2}-\left(k_{n} L\right)^{2}\right]\left[\beta_{l}^{2}-\left(k_{n} L\right)^{2}\right]}, \\
& I_{4,1}=\frac{\left(\beta_{l} \sin \beta_{l}+i k_{n} L \cos \beta_{l}\right) e^{\beta_{j}}-i k_{n} L e^{\beta_{j}-i k_{n} L}}{\left(\beta_{j}-i k_{n} L\right)\left[\beta_{l}^{2}-\left(k_{n} L\right)^{2}\right]}-\frac{\left(\beta_{l} \sin \beta_{l}-i k_{n} L \cos \beta_{l}\right) e^{-i k_{n} L}+i k_{n} L}{\left(\beta_{j}+i k_{n} L\right)\left[\beta_{l}^{2}-\left(k_{n} L\right)^{2}\right]}-\frac{2 i k_{n} L}{\beta_{j}^{2}+\left(k_{n} L\right)^{2}} \\
& \times\left[\frac{\left(\beta_{l} \sin \beta_{l}+\beta_{j} \cos \beta_{l}\right) e^{\beta_{j}}-\beta_{j}}{\beta_{j}^{2}+\beta_{l}^{2}}\right], \\
& I_{4,3}=\frac{\beta_{j}\left[\left(\beta_{l} \sin \beta_{l}-i k_{n} L \cos \beta_{l}\right) e^{-i k_{n} L}+i k_{n} L\right]}{\left[\beta_{j}^{2}-\left(k_{n} L\right)^{2}\right]\left[\beta_{l}^{2}-\left(k_{n} L\right)^{2}\right]}+\frac{i k_{n} L}{\beta_{j}^{2}-\left(k_{n} L\right)^{2}}\left[\frac{1-\cos \left(\beta_{j}+\beta_{l}\right)}{\beta_{j}+\beta_{l}}+\frac{1-\cos \left(\beta_{j}-\beta_{l}\right)}{\beta_{j}-\beta_{l}}\right] \\
& -\frac{\left(i k_{n} L \sin \beta_{j}+\beta_{j} \cos \beta_{j}\right)\left[\left(\beta_{l} \sin \beta_{l}+i k_{n} L \cos \beta_{l}\right)-i k_{n} L e^{-i k_{n} L}\right]}{\left[\beta_{j}^{2}-\left(k_{n} L\right)^{2}\right]\left[\beta_{l}^{2}-\left(k_{n} L\right)^{2}\right]}, \\
& I_{4,4}=\frac{\left(\beta_{j} \sin \beta_{j}-i k_{n} L \cos \beta_{j}\right)\left[\left(\beta_{l} \sin \beta_{l}+i k_{n} L \cos \beta_{l}\right)-i k_{n} L e^{-i k_{n} L}\right]}{\left[\beta_{j}^{2}-\left(k_{n} L\right)^{2}\right]\left[\beta_{l}^{2}-\left(k_{n} L\right)^{2}\right]}+\frac{i k_{n} L}{\beta_{j}^{2}-\left(k_{n} L\right)^{2}}\left[\frac{\sin \left(\beta_{j}+\beta_{l}\right)}{\beta_{j}+\beta_{l}}+\frac{\sin \left(\beta_{j}-\beta_{l}\right)}{\beta_{j}-\beta_{l}}\right] \\
& -\frac{i k_{n} L\left[\left(\beta_{l} \sin \beta_{l}-i k_{n} L \cos \beta_{l}\right) e^{-i k_{n} L}+i k_{n} L\right]}{\left[\beta_{j}^{2}-\left(k_{n} L\right)^{2}\right]\left[\beta_{l}^{2}-\left(k_{n} L\right)^{2}\right]} \text {. }
\end{aligned}
$$

The remaining seven components are determined based on the above results. $I_{1,2}$ is obtained by replacing $\beta_{j}$ with $\left(-\beta_{j}\right)$ in Eq. (A1); $I_{2,1}$ is obtained by replacing $\beta_{l}$ with $\left(-\beta_{l}\right)$ in Eq. (A1); $I_{2,2}$ is obtained by replacing $\beta_{j}$ and $\beta_{l}$ with $\left(-\beta_{j}\right)$ and $\left(-\beta_{l}\right)$, respectively, in Eq. (A1); $I_{2,3}$ is obtained by replacing $\beta_{l}$ with $\left(-\beta_{l}\right)$ in Eq. (A2); $I_{2,4}$ is obtained by replacing $\beta_{l}$ with $\left(-\beta_{l}\right)$ in Eq. (A3); $I_{3,2}$ is obtained by replacing $\beta_{j}$ with $\left(-\beta_{j}\right)$ in Eq. (A4); and $I_{4,2}$ is obtained by replacing $\beta_{j}$ with $\left(-\beta_{j}\right)$ in Eq. (A7). When $j=l$, four items in Eqs. (A1)-(A9) become $0 / 0$ type and should be replaced by the finite values given below:

$$
\begin{aligned}
& \frac{1-e^{\beta_{l}-\beta_{j}}}{\beta_{j}-\beta_{l}}=1, \quad \frac{e^{\beta_{j}-\beta_{l}}-1}{\beta_{j}-\beta_{l}}=1, \\
& \frac{\sin \left(\beta_{j}-\beta_{l}\right)}{\beta_{j}-\beta_{l}}=1, \quad \frac{1-\cos \left(\beta_{j}-\beta_{l}\right)}{\beta_{j}-\beta_{l}}=0 .
\end{aligned}
$$

Note that for frequency below the first cut-on of the duct, only the plane wave mode, $n=0$, has real wave number $k_{0}$, and there are chances that $k_{0} L \rightarrow \beta_{j}$ and/or $\beta_{l}$. In such case, some items in Eqs. (A1)-(A9) will also become 0/0 type. This problem can be avoided numerically easily, hence, it is not addressed here.

The $I_{c j, p}$, and $I_{c l, q}(p, q$ from 1 to 4$)$ are given below:

$$
\begin{gathered}
I_{c j, 1}=\frac{\beta_{j}\left[\cos (m \pi) e^{\beta_{j}}-1\right]}{\beta_{j}^{2}+(m \pi)^{2}}, \quad I_{c j, 2}=\frac{\beta_{j}\left[1-\cos (m \pi) e^{-\beta_{j}}\right]}{\beta_{j}^{2}+(m \pi)^{2}}, \\
I_{c j, 3}=\frac{\beta_{j}\left[1-\cos \left(\beta_{j}+m \pi\right)\right]}{\beta_{j}^{2}-(m \pi)^{2}}, \quad I_{c j, 4}=\frac{\beta_{j} \sin \left(\beta_{j}+m \pi\right)}{\beta_{j}^{2}-(m \pi)^{2}},
\end{gathered}
$$$$
I_{c l, 1}=\frac{\beta_{l}\left[\cos (m \pi) e^{\beta_{l}}-1\right]}{\beta_{l}^{2}+(m \pi)^{2}}, \quad I_{c l, 2}=\frac{\beta_{l}\left[1-\cos (m \pi) e^{\left.-\beta_{l}\right]}\right.}{\beta_{l}^{2}+(m \pi)^{2}},
$$ 
$I_{c l, 3}=\frac{\beta_{l}\left[1-\cos \left(\beta_{l}+m \pi\right)\right]}{\beta_{l}^{2}-(m \pi)^{2}}, \quad I_{c l, 4}=\frac{\beta_{l} \sin \left(\beta_{l}+m \pi\right)}{\beta_{l}^{2}-(m \pi)^{2}}$.

(A11)

Ashby, M. F. (2005). Material Selection in Mechanical Design, 3rd ed. (Butterworth Heinemann, Oxford).

Brown, S. (1964). "Acoustic design of broadcasting studios," J. Sound Vib. 1, 239-257.

Choy, Y. S., and Huang, L. (2002). "Experimental studies of a drumlike silencer," J. Acoust. Soc. Am. 112, 2026-2035.

Choy, Y. S., and Huang, L. (2005). "Effect of flow on the drumlike silencer," J. Acoust. Soc. Am. 118, 3077-3085.

Doak, P. E. (1973). "Excitation, transmission and radiation of sound from source distributions in hard-walled ducts of finite length. I. The effects of duct cross-section geometry and source distribution space-time pattern," J. Sound Vib. 31, 1-72.

Fernyhough, M., and Evans, D. V. (1996). "Comparison of a step approximation to an exact solution of acoustic scattering in a uniform-width pipe with non-uniform wall impedance," Q. J. Mech. Appl. Math. 49, 419437.

Ford, R. D., and McCormick, M. A. (1969). "Panel sound absorbers," J. Sound Vib. 10, 411-423.

Frommhold, W., Fuchs, H. V., and Sheng, S. (1994). "Acoustic performance of membrane absorbers," J. Sound Vib. 170, 621-636.

Grant, A. D., and Lawrie, J. B. (2000). "Propagation of fluid-loaded struc- tural waves along a duct with smoothly varying bending characteristics," Q. J. Mech. Appl. Math. 53, 299-321.

Horoshenkov, K. V., and Sakagami, K. (2001). "A method to calculate the acoustic response of a thin, baffled, simply supported poroelastic plate," J. Acoust. Soc. Am. 110, 904-917.

Huang, L. (2001). "A theoretical study of passive control of duct noise using panels of varying compliance," J. Acoust. Soc. Am. 109, 2805-2814.

Huang, L. (2002). "Modal analysis of a drumlike silencer," J. Acoust. Soc. Am. 112, 2014-2025.

Huang, L. (2004). "Parametric studies of a drumlike silencer," J. Sound Vib. 269, $467-488$.

Huang, L. (2006). "Broadband sound reflection by plates covering sidebranch cavities in a duct," J. Acoust. Soc. Am. 119, 2628-2638.

Ingard, K. U. (1994). "Notes on the sound absorption technology," Noise Control Foundation, Poughkeepsie, NY.

Inman, D. J. (2001). Engineering Vibration (Prentice Hall, Englewood Cliffs, N.J)

Kuttruff, H. (2000). Room Acoustics (E \& FN Spon, New York).

Mechel, F. P., and Vér, I. L. (1992). Noise and Vibration Control Engineering: Principles and Applications, edited by L. L. Beranek and I. L. Vér (Wiley, New York), Chap. 8.

Munjal, M. L., and Doige, A. G. (1990). "Theory of a two source-location method for direct experimental evaluation of the four-pole parameters of an aeroacoustic element," J. Sound Vib. 141, 323-333.

Sakagami, K., Kiyama, M., Morimoto, M., and Takahashi, D. (1996). "Sound absorption of a cavity-backed membrane: A step towards design method for membrane-type absorber," Appl. Acoust. 49, 237-247. 